\title{
Recent Advancements in the Loading and Modification of Therapeutic Exosomes
}

\begin{abstract}
Mengqiao $\mathrm{Xu}^{1,2,3,4,5 t}$, Qianhao Yang ${ }^{6 \dagger}$, Xiaodong Sun ${ }^{1,2,3,4,5 *}$ and Yue Wang ${ }^{7,8 *}$
${ }^{1}$ Shanghai General Hospital, Shanghai, China, ${ }^{2}$ National Clinical Research Center for Eye Diseases, Shanghai, China, ${ }^{3}$ Shanghai Key Laboratory of Ocular Fundus Diseases, Shanghai, China, ${ }^{4}$ Shanghai Engineering Center for Visual Science and Photomedicine, Shanghai, China, ${ }^{5}$ Shanghai Engineering Center for Precise Diagnosis and Treatment of Eye Diseases, Shanghai, China, ' Department of Orthopedic Surgery, Shanghai Jiao Tong University Affiliated Sixth People's Hospital, Shanghai, China, ${ }^{7}$ Department of Histology and Embryology, Second Military Medical University, Shanghai, China,

${ }^{8}$ Shanghai Key Lab of Cell Engineering, Shanghai, China
\end{abstract}

Exosomes have a rapid development of bio-nanoparticles for drug delivery and confluent advances in next-generation diagnostics, monitoring the progression of several diseases, and accurate guidance for therapy. Based on their prominent stability, cargo-carriage properties, stable circulating capability, and favorable safety profile, exosomes have great potential to regulate cellular communication by carrying variable cargoes into specific site. However, the specific loading strategies and modification methods for engineered exosomes to enhance the targeting ability are unclear. The clinical application of exosomes is still limited. In this review, we discuss both original and modified exosomes for loading specific therapeutic molecules (proteins, nucleic acids, and small molecules) and the design strategies used to target specific cells. This review can be used as a reference for further loading and modification strategies as well as for the therapeutic applications of exosomes.

Keywords: exosome, drug delivery, engineering strategy, content loading, surface modification

\section{INTRODUCTION}

tThese authors have contributed equally to this work and share first authorship

Specialty section:

This article was submitted to Bionics and Biomimetics,

a section of the journal Frontiers in Bioengineering and Biotechnology

Received: 22 July 2020 Accepted: 16 September 2020 Published: 11 November 2020

Citation:

Xu M, Yang $Q$, Sun X and Wang Y (2020) Recent Advancements in the Loading and Modification of Therapeutic Exosomes. Front. Bioeng. Biotechnol. 8:586130. doi: 10.3389/fbioe.2020.586130
The past decade has witnessed the rapid development of bio-nanoparticles and confluent advances in next-generation diagnostics, monitoring the progression of several diseases, and accurate guidance for therapy (Chen G. et al., 2018; Tran et al., 2019). The research on cell-secreted extracellular vehicles (EVs), such as bio-nanoparticles, has expanded exponentially. As the main classes of EVs secreted into extracellular, exosomes play essential roles in cell-cell, cell-tissue and cross-species communication (Zhou et al., 2017), and these subjects are covered in this review.

Exosomes are specialized membranous 30-150 nm EVs released from multiple cells upon membrane fusion and harvested through body fluids or cell culture (Liew et al., 2017). Based on their prominent stability, cargo-carriage properties, stable circulating capability, and favorable safety profile, exosomes have great potential to regulate cellular communication by carrying variable cargoes into specific site (Liu and Su, 2019). Exosomes could easily across diversified barriers containing many therapeutic drugs, especially the blood/brain barriers (Bonoiu et al., 2009; De Rosa et al., 2012). The cargos in exosomes are efficiently delivered into the cytoplasm with minimal induced toxicity and include proteins, nucleic acids, and chemicals linked to the pathogenesis of many diseases (Bunggulawa et al., 2018). Moreover, the unique mechanism of targeting to the derived cells of exosomes were also highlighted, as a promising drug delivery vehicles (Park et al., 2019). 
Despite the ever-deeper understanding of nanomedicine, much strategies remains to be improved before exosomes can be successfully applied for clinical applications, such as intracellular communication, immune system development, and neuron cell signaling (Alenquer and Amorim, 2015). The standard techniques for the characterization, separation, and storage need substantial development to better apply exosomes in the clinic. On the other hand, some side effects, such as transferred drug resistance or inhibited immune responses, also need to be considered to avoid deleterious outcomes. Various modified strategies of exosome generation through chemical methods or genetic engineering may overcome these barriers to improve the carrying capacity and specificity for a better therapeutic effect. This review can be used for further development of loading and modification strategies as well as for the therapeutic applications of engineered exosomes.

\section{THE CONTEXT LOADING STRATEGIES OF EXOSOMES FOR VARIOUS SUBSTANCE DELIVERY}

An exosome or exosome mimetics-based delivery system has desirable benefits that make it a superior choice, such as specificity to target tissues, stability, a long-circulating halflife, and biocompatibility with minimal toxic effects. Exosomes could transport different cargos to targeting tissues rapidly with high stability through their homing characteristics (Figure 1 and Table 1). As exosomes are a small and natural product, they can avoid immune responses, such as membrane fusion and phagocytosis, thus bypassing engulfment by lysosomes. The exosome can also be loaded with multiple water-soluble drugs because of its hydrophilic core.

\section{The Engineering Strategies of Exosomes Loading With Proteins}

The sufficient protein supplement is essential for the normal physiological process. For example, the deficiency of specific tumor suppressors is the leading cause of malignant tumors. Competent drug delivery vectors have great potential to accurately transporting therapeutic cargoes. The biological performance of exosomes has been extensively researched nowadays. The major hurdle has been the adequate loading of the desired protein into exosomes with minimum influence to the cell membrane and bioactivity of cargoes. Currently, several methods have been listed for exosomal loading to target specific tissues.

\section{Overexpression of Cytoplasmic Proteins}

The genetic engineering procedure for loading exosomes is conducted by transfection specific genes of interest into donor cells. Protein synthesis is modulated by the inserted genes, and then, proteins are secreted in exosomes through natural packing processes. After isolation and purification, engineered exosomes can be obtained (Hall et al., 2016).

Although this approach seems feasible and straightforward, there are many problems to be resolved. The proliferation and apoptosis of donor cells might be imbalanced due to overexpressed protein. A sophisticated sorting system should be used to overcome challenges of non-specific target proteins being loaded with high efficiency in exosomes.

\section{Fusion Proteins Targeted to Exosomes}

Several strategies have been designed to explicitly load target proteins. Di Bonito et al. have used dedicated DNA vector, which could express the fusion protein combining HPV-E7 and Nef exosome-anchoring protein. Fusion proteins that are not degraded and externally neutralized can be delivered into exosomes, which improves the specificity and loading efficiency of targeted proteins in exosomes (Manfredi et al., 2016).

\section{Reduction in the Permeabilization of the Exosome Membrane}

The challenges to developing catalase as a therapeutic agent were caused by the rapid metabolism of catalase and the penetration across blood-brain barrier (BBB). Batrakova and coworkers reported the new strategy to deliver catalase as the specific treatment for neuronal disease (Haney et al., 2015). After isolated from RAW 264.7 mouse macrophages, the exosomes loaded with catalase could easily transferred across multiple barriers with saponin, as a brand-new strategy reducing the permeabilization, compared with previous loading methods. The incredible neuroprotective effects could be easily achieved after intranasal administration of the exosomes (Haney et al., 2015).

\section{Intrinsic Modification of the Target Protein}

As a natural transporter of antigens, exosomes could efficiently carry certain vaccine for many treatments. Ubiquitin-fused target proteins were originated from human embryonic kidney (HEK 293) cells. After the protein was fused to ubiquitin, the concentration of the target protein was increased approximately ten-fold in the exosomes. Additionally, the immunoreaction of $\mathrm{T}$ cell might be caused by the recombinant antigen from the exosomes (Cheng and Schorey, 2016).

The ubiquitination of targeted protein has also been applied as an effective loading strategy by Sterzenbach. Cre recombinase was designed for ubiquitination, labeled with WW tag and can be easily recognized by late-domain (L-domain) pathway (Sterzenbach et al., 2017). Therefore, protein ubiquitination provides a specific signal for protein delivery and loading into exosomes. However, the degradation and dysfunction of the modified protein still need to be considered.

\section{Various Mechanical Methods}

Several mechanical methods, including mechanical extrusion, saponin permeabilization, incubation at room temperature, repeated freeze-thaw cycles, and sonication were verified by Haney et al. for loading of catalase to treat Parkinson's disease (PD). The overexpressed cytoplasmic protein combined with mechanical selection such as sonication, saponin permeabilization, and mechanical extrusion were highly efficient, protecting the catalase from degradation and maintaining a sustained release. Then, the exosomes were efficiently loaded and internalized for the treatment of neuronal disease (Haney et al., 2015). 


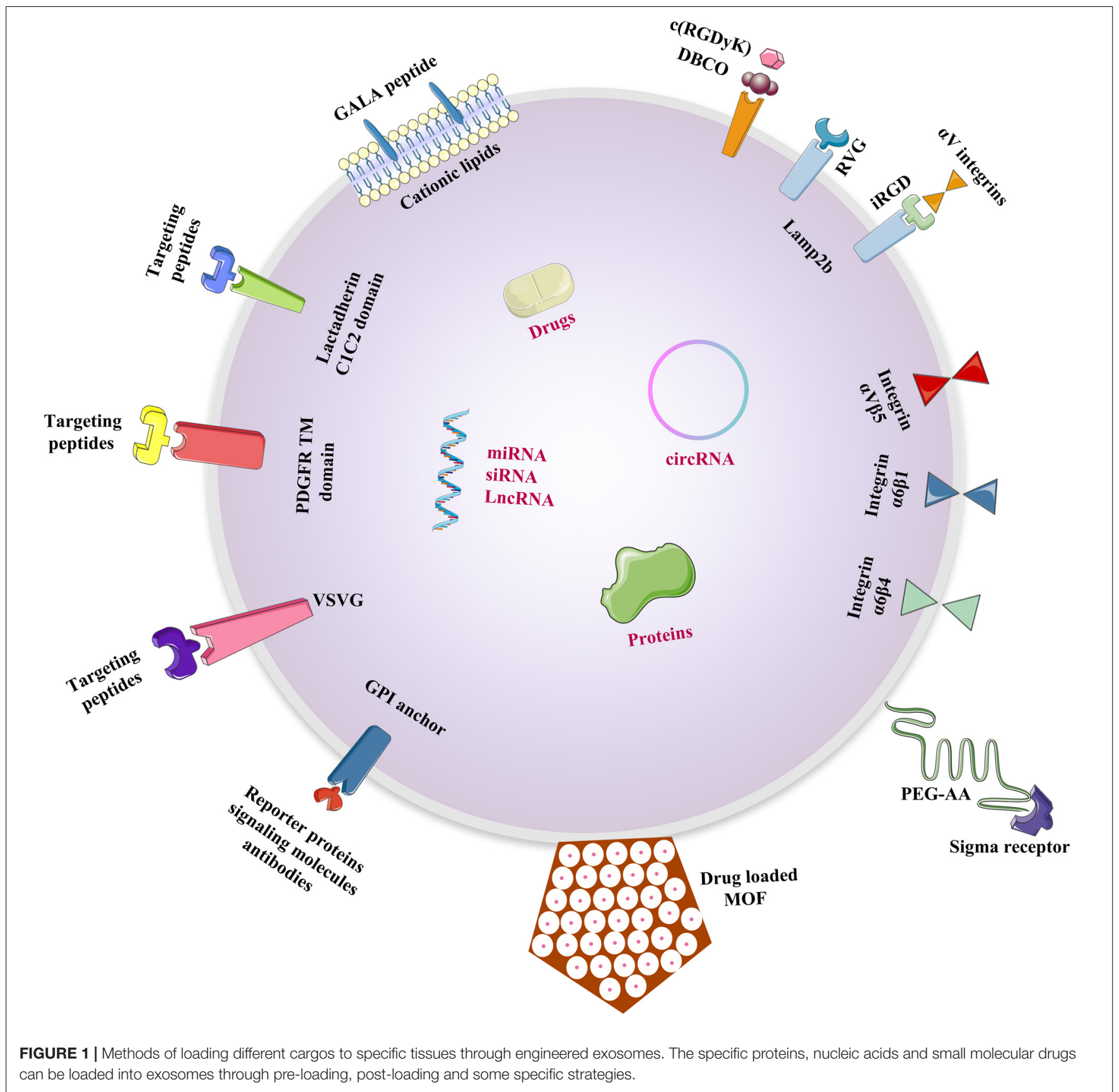

There are still some technical difficulties to be resolved. The purification of exosomes remains laborious, and the integrity and biological activity of exosomes are incompletely resolved to date. The composition of the mechanically generated exosomes might be different with cell-generated exosomes with low efficiency.

\section{The Engineering Strategies of Exosomes Loading With Nucleic Acids}

The sufficient supplement of DNA, RNA and various nucleic acids is essential for the normal physiological process. Exosome has been widely used for the development of genetic therapy, as an effective delivery strategy for the genetic materials. Nowadays, several methods have been listed for exosomal loading to target specific tissues through the alteration of gene and protein expression.

\section{Transfection With Different Reagents}

After the isolation of exosomes from HeLa cells, the mixture of short interfering RNA (siRNA) AF488 and lipofectamine was incubated with exosomes at room temperature (RT) for $30 \mathrm{~min}$. The exosomes loaded with siRNA were then transferred to recipient cells after being in culture for $24 \mathrm{~h}$ (Shtam et al., 2013; Li et al., 2018). 
TABLE 1 | Different methods in existing studies of loading specific proteins, nucleic acids and small molecular drugs into engineered exosomes.

\begin{tabular}{|c|c|c|c|c|c|c|}
\hline Delivery cargo & Exosome source & $\begin{array}{l}\text { Isolation } \\
\text { methodology }\end{array}$ & $\begin{array}{l}\text { Loading } \\
\text { methodology }\end{array}$ & Therapeutic effect & Efficiency & References \\
\hline \multicolumn{7}{|l|}{ Proteins } \\
\hline \multirow[t]{4}{*}{ Catalase } & Raw 264.7 macrophages (mouse) & Ultracentrifugation & Mixing & $\begin{array}{l}\text { Preserve catalase enzymatic activity, prolong } \\
\text { blood circulation time, and reduce } \\
\text { immunogenicity, thereby improve drug } \\
\text { therapeutic efficacy of many CNS disease. }\end{array}$ & $4.9(\mathrm{SEM} \pm 0.5 \%)$ & $\begin{array}{l}\text { Haney et al., 2015; } \\
\text { Zarovni et al., } 2015\end{array}$ \\
\hline & & & $\begin{array}{l}\text { Transgenic mice } \\
\text { with loxP-tdTomato }\end{array}$ & & $18.5(\mathrm{SEM} \pm 1.3 \%)$ & \\
\hline & & & Sonication & & 26.1 (SEM $\pm 1.2 \%)$ & \\
\hline & & & Extrusion & & $22.2(\mathrm{SEM} \pm 3.1 \%)$ & \\
\hline Cre recombinase & LN18 & Ultracentrifugation & Transfection & $\begin{array}{l}\text { identifying functional delivery of exosomes } \\
\text { across blood-brain barrier to recipient neurons } \\
\text { in the brain. }\end{array}$ & / & $\begin{array}{l}\text { Sterzenbach et al., } \\
2017\end{array}$ \\
\hline BDNF & Raw 264.7 macrophages (mouse) & Ultracentrifugation & Incubation & $\begin{array}{l}\text { The delivery is enhanced in the presence of } \\
\text { brain inflammation, a condition often presents } \\
\text { in CNS diseases. }\end{array}$ & $\sim 20 \%$ & Yuan et al., 2017 \\
\hline \multicolumn{7}{|l|}{ Nucleic acid } \\
\hline BACE1 SiRNA & Dendritic cells & Ultracentrifugation & Electroporation & $\begin{array}{l}\text { Specific gene knockdown after specific siRNA } \\
\text { delivery to the brain for Alzheimer's disease }\end{array}$ & $\sim 20 \%$ & $\begin{array}{l}\text { Alvarez-Erviti et al., } \\
2011\end{array}$ \\
\hline$B C R-A B L$ siRNA & HEK293T & Ultracentrifugation & Transfection & $\begin{array}{l}\text { overcome pharmacological resistance in } \mathrm{CML} \\
\text { cells }\end{array}$ & / & Bellavia et al., 2017 \\
\hline$K R A S^{G 12 D}$ siRNA & Mouse fibroblasts & Ultracentrifugation & Electroporation & $\begin{array}{l}\text { Suppression of tumor growth in pancreatic } \\
\text { cancer }\end{array}$ & / & $\begin{array}{l}\text { Kamerkar et al., } \\
2017\end{array}$ \\
\hline MAPK siRNA & Plasma (human) & Ultracentrifugation & Electroporation & $\begin{array}{l}\text { The MAPK- } 1 \text { was down-regulated in } \\
\text { monocytes and lymphocytes }\end{array}$ & / & $\begin{array}{l}\text { Wahlgren et al., } \\
2012\end{array}$ \\
\hline RAD51 and RAD52 siRNA & Malignant ascites fluid (mouse) & Ultracentrifugation & $\begin{array}{l}\text { Mixing with } \\
\text { lipofectamine }\end{array}$ & $\begin{array}{l}\text { causing post-transcriptional gene silencing and } \\
\text { massive reproductive cell death in recipient } \\
\text { cells. }\end{array}$ & // & Shtam et al., 2013 \\
\hline GAPDH SiRNA & Primary immature Dendritic cells & Ultracentrifugation & Electroporation & $\begin{array}{l}\text { Specific gene knockdown after specific siRNA } \\
\text { delivery to the brain for Alzheimer's disease }\end{array}$ & $10-38 \%$ & $\begin{array}{l}\text { Alvarez-Erviti et al., } \\
2011\end{array}$ \\
\hline VEGF SiRNA & Dendritic cells & Ultracentrifugation & Electroporation & Suppression of tumor growth in breast cancer. & $3 \%$ & Wang et al., 2017 \\
\hline Let-7a mimic & HEK293 & Ultracentrifugation & Transfection & $\begin{array}{l}\text { therapeutically to target EGFR-expressing } \\
\text { cancerous tissues with nucleic acid drugs for } \\
\text { breast cancer. }\end{array}$ & / & Ohno et al., 2013 \\
\hline Cas9/sgRNA & SKOV-3 & ExoQuick & Electroporation & $\begin{array}{l}\text { suppressing expression of poly (ADP-ribose) } \\
\text { polymerase-1 (PARP-1), resulting in the } \\
\text { induction of apoptosis in ovarian cancer }\end{array}$ & $\sim 1.75 \%$ & Kim et al., 2017 \\
\hline miRNA & Glioblastoma cells & Ultracentrifugation & Transfection & Providing diagnostic information & I & Skog et al., 2008 \\
\hline
\end{tabular}


TABLE 1 | Continued

\begin{tabular}{|c|c|c|c|c|c|c|}
\hline Delivery cargo & Exosome source & $\begin{array}{l}\text { Isolation } \\
\text { methodology }\end{array}$ & $\begin{array}{l}\text { Loading } \\
\text { methodology }\end{array}$ & Therapeutic effect & Efficiency & References \\
\hline & $\begin{array}{l}\text { Human cord blood endothelial } \\
\text { colony-forming cells }\end{array}$ & Ultracentrifugation & & $\begin{array}{l}\text { Protected kidney function and reduced kidney } \\
\text { injury }\end{array}$ & / & Vinas et al., 2016 \\
\hline \multicolumn{7}{|c|}{ Small-molecule drugs } \\
\hline \multirow[t]{6}{*}{ Paclitaxel } & Raw 264.7 & ExoQuick & Sonication & $\begin{array}{l}\text { Overcome MDR cancer and Reduced } \\
\text { pulmonary metastases in vitro and in vivo }\end{array}$ & 28.29 (SEM $\pm 1.38 \%)$ & Kim et al., 2016 \\
\hline & & & Mixing & & $1.4(\mathrm{SEM} \pm 0.38 \%)$ & \\
\hline & & & Electroporation & & $5.3(\mathrm{SEM} \pm 0.48 \%)$ & \\
\hline & Milk & Ultracentrifugation & Incubation & Oral & $7.9(\mathrm{SEM} \pm 1.0 \%)$ & Agrawal et al., 2017 \\
\hline & LNCaP and PC-3 (human) & Ultracentrifugation & Mixing & $\begin{array}{l}\text { Enhanced drug cytotoxicity to prostate cancer } \\
\text { cells }\end{array}$ & $9.2(\mathrm{SD} \pm 4.5 \%)$ & Saari et al., 2015 \\
\hline & Mesenchymal stromal cells & Ultracentrifugation & Incubation & $\begin{array}{l}\text { Inhibited growth of human pancreatic } \\
\text { adenocarcinoma cell }\end{array}$ & / & $\begin{array}{l}\text { Pascucci et al., } \\
2014\end{array}$ \\
\hline \multirow[t]{4}{*}{ Doxorubicin } & $\begin{array}{l}\text { Immature dendritic Cells transfected } \\
\text { with the vector expressing } \\
\text { iRGD-Lamp2b fusion proteins }\end{array}$ & Ultracentrifugation & Electroporation & $\begin{array}{l}\text { Specific drug delivery to the tumor site and } \\
\text { inhibited tumor growth }\end{array}$ & $<20 \%$ & Tian et al., 2014 \\
\hline & Reticulocytes & $\begin{array}{l}\text { Magnetic } \\
\text { separation }\end{array}$ & Incubation & & & \\
\hline & LIM1215 & Ultracentrifugation & & & & \\
\hline & Raw 264.7 & ExoQuick & Sonication & & $8.0-11.0 \%$ & Lee et al., 2018 \\
\hline \multirow[t]{2}{*}{ Curcumin } & $\begin{array}{l}\text { Mouse lymphoma cell (EL-4) and RAW } \\
264.7 \text { cells }\end{array}$ & Ultracentrifugation & Mixing & Enhanced anti-inflammatory activity & / & Sun et al., 2010 \\
\hline & $\begin{array}{l}\text { Tumor cells (GL26-Luc, BV2, 3T3L1, } \\
4 \mathrm{~T} 1, \mathrm{CT} 26, \mathrm{~A} 20 \text {, and EL-4) }\end{array}$ & & & $\begin{array}{l}\text { Inhibited brain inflammation and delayed brain } \\
\text { tumor growth }\end{array}$ & & Zhuang et al., 2011 \\
\hline Dopamine & Kunming mice blood & Ultracentrifugation & Incubation & $\begin{array}{l}\text { Enhanced therapeutic effect due to } \\
\text { brain-specific drug delivery }\end{array}$ & / & Qu et al., 2018)0 \\
\hline
\end{tabular}




\section{Electroporation}

The concept of engineered exosomes to deliver bioactive molecules was first realized by Alvarez-Erviti et al. (2011). After loading with small interference (si) RNA, with the successful fusion of membrane protein Lamp2b of exosomes and rabies viral glycoprotein (RVG) peptide, purified exosomes from dendritic cells could target the to neuronal cells for subsequentially therapy. The RVG-linked exosomes were then injected intravenously with GAPDH siRNA to verify the capability and feasibility of their transport across the blood-brain barrier (BBB), as well as the effective GAPDH knockdown. BACE1-targeted siRNA and $\mathrm{KRAS}^{G 12 D}$ siRNA were also loaded into exosomes by electroporation for treating Alzheimer's disease and suppressing tumor growth (Alvarez-Erviti et al., 2011; Kamerkar et al., 2017).

The abovementioned direct methods, such as loading with transfection reagents or electroporation, require repeated separation and purification. Moreover, multiple methods have been used for the purification of exosomes, including differential ultracentrifugation, size-based separation, and exosome precipitation (Heinemann and Vykoukal, 2017; Niu et al., 2017). Repeated purification and high-speed centrifugation might result in the loss of exosomes and/or a reduction in sample quality.

\section{Guidance of Proteins, Peptides or Signature Sequences}

New technology has also been developed based on the abovementioned disadvantages, including the RNA packaging device for mRNA delivery to exosomes and to target cells. The method is relatively efficient and does not require a high concentration of exosomes. The mRNA of catalase was readily delivered into the brain by engineered exosomes, where it significantly reduced neuroinflammation and neurotoxicity (Kojima et al., 2018). The protein L7Ae combined with C/D box were used as the RNA-packaging device (Saito et al., 2010). The gap junction protein Cx43 was also applied for the cytoplasmic transfer. With these tools, various mRNAs loaded with engineered exosomes could efficiently transfer to the target cells.

The enriched RNAs (eRNAs) has also been applied for RNA targeting in exosomes. The three motifs with conserved sequences were found in an analysis of RNAs in exosomes. Therefore, the signature sequences in these studies could be used as the loading strategy for extended therapy (Batagov et al., 2011).

\section{Transfection}

The protein packaging delivery methods described above could also be applied to miRNAs, which are naturally secreted into exosomes upon transfection into parental cells.

After transfected with let-7a, as a specific miRNA for tumor suppression, engineered exosomes were intravenously administered to suppress tumor growth by Ohno et al. (2013). Moreover, miR-124, miR-126, and HGF siRNA derived from cells of various origins were also transfected. The obtained exosomes containing these nucleic acids were verified for different therapeutic effects on cancer cells and myocardial injury (Luo et al., 2017; Wang et al., 2018; Zhang et al., 2018). However, the cytotoxicity, inefficient packaging, and poor specificity still need to be solved.

\section{The Powerful Tool of CRISPR/Cas9 for Generating Exosome-Liposome Hybrids}

As an effective and popular tool for genomic studies, CRISPR/Cas9 could precisely alter the DNA sequence of the target gene by a single guide RNA (sgRNA). For example, Cas9 and sgRNA targeting poly(ADP-ribose) polymerase 1 (PARP-1) have been applied for the engineered exosomes by electroporation, while the loading efficiency for such macromolecular nucleic acids is relatively lower (Lamichhane et al., 2015; Kim et al., 2017). Therefore, hybrid exosomeliposome nanovesicles were designed by Tan et al. using the pure incubation method to deliver CRISPR-Cas9-expressing vectors more efficiently. These hybrid nanoparticles were endocytosed and efficiently suppress relative gene expression in mesenchymal stem cells (MSCs) (Lin et al., 2018). The promising application of this exciting approach in gene manipulation awaits further evaluation.

In summary, despite the extensive use of electroporation, some RNAs with unique structures, such as modified miRNAs, shRNAs, mRNAs, or RNAs, are not suitable for exosomes used in clinical application. The EXOtic devices for specific mRNAs and eRNAs have significantly enhanced loading efficiency for further application.

\section{The Engineering Strategies of Exosomes Loading With Small-Molecule Cargo}

Extensive research has demonstrated that exosomes act as vehicles to deliver chemotherapy drugs for therapeutic application. The loading methods include sonication, direct mixing, incubation, and eddy current oscillation.

Paclitaxel (PTX) is not commonly used in the clinic for solid tumors due to its exceedingly poor aqueous solubility, but it can be loaded into exosomes by multiple cycles of sonication with higher efficiency than has been achieved with other methods, such as incubation, saponin permeabilization, freezing and thawing, or extrusion (Haney et al., 2015; Kim et al., 2016). The administration of exosomal paclitaxel was further evaluated and found to exert significant antimetastatic efficacy and superior survival ability for metastatic tumor growth compared to Taxol (Kim et al., 2018). Moreover, the exosomes were also loaded with paclitaxel by the pure incubation method with increasing drug loading efficacy to form orally administered exosomal paclitaxel. Electrical perforation and ultrasonic treatment were also applied as the loading strategy with lower efficiency.

Moreover, iRGD exosomes and reticulocyte (RTC)-derived exosomes were designed for loading with doxorubicin by electroporation and moderate stirring to improve the payload efficiency to the tumor tissue (Tian et al., 2014; Qi et al., 2016). These passive loading methods are widely used for the loading of small molecule drugs; however, the degradation and loss of exosomes remains unabated in the process of purification. Moreover, the bioactivity and stability of these exosomes are significantly affected upon prolonged treatment and physicochemical properties. Therefore, the stability of 
exosomes, proper storage conditions and more loading strategies are worthy of study.

\section{SURFACE MODIFIED EXOSOMES FOR VARIOUS SUBSTANCE DELIVERY}

Currently, confluent advances have been made with respect to bio-nanoparticles in terms of next-generation diagnostics, disease surveillance, and individual diagnosis and therapy. Despite everdeeper understanding, the advances of modification of exosomes are essential for the improvement of the clinical translation.

The delicate drug delivery strategy targeting to a particular tissue or a specific type of cell could avoid decentralized distribution to other tissues and preventing degradation by immune responses (Barile and Vassalli, 2017). Exosomes exhibited excellent biocompatibility and low-level long-term with natural origin compared with those of other DDSs. Therefore, modifications to the exosomal surface and components of the donor cells need to be studied for the improvement of targeting strategies (Figure 2).

\section{Surface-Modified Exosomes for Cerebral Ischemia Therapy}

After inserting targeting proteins into donor cells, exosomes containing these targeting proteins are secreted and can be harvested and used by target antigens. Recently, the lactadherin C1C2 domain combined and Lamp2b were both used for accurate targeting.

In addition, $\alpha \mathrm{V}$ integrins can be recognized explicitly by iRGD, which was loaded onto exosomes targeting tumor cells for the treatment of malignant tumors. The large amounts of exosomes from immature dendritic cells (imDCs) with an overexpressed fusion protein of iRGD and Lamp2b might be obtained by purification carried out with iRGD-targeting proteins. The imDC-exosomes loaded with doxorubicin by electroporation were specifically targeted to breast cancer cells, and they showed a direct therapeutic role in breast cancer mouse models (Tian et al., 2014).

Specific surface modification can also enhance the targeting ability of exosomes for cerebral ischemic therapy. The cyclo(ArgGly-Asp-DTyr-Lys) peptide $[\mathrm{c}(\mathrm{RGDyK})]$ and rabies virus glycoprotein (RVG) were used for explicit targeting to the brain. Tian et al. proposed that engineered $\mathrm{c}(\mathrm{RGDyK})$ exosomes loaded with curcumin, accumulated in considerable amounts in ischemic brain lesions following intravenous administration. Through chemical conjugation, bioorthogonal copper-free azidealkyne cycloaddition was applied for generating mesenchymal stromal cell (MSC)-derived exosomes for integrin $\alpha \mathrm{v} \beta 3$ precisely located in ischemic tissue (Tian et al., 2018). Modified exosomes expressing specific RVG peptides could efficiently deliver miR-124 and opioid receptor (MOR) siRNA to a specific site in the brain, exhibiting accurate treatment for brain infarction and prevention of morphine relapse (Liu et al., 2015; Yang et al., 2017).

It is of great potential to artificially design the modification the membranes of donor cells and exosomes, thus targeting the specific tissues. Nanobodies that anchor glycosylphosphatidylinositol (GPI) on the surfaces of EVs can exhibit multiple proteins, such as reporter proteins, signaling molecules, and antibodies.

\section{Exosomes Combined With pH-Sensitive Fusogenic Peptides and Cationic Lipids for Cytosolic Delivery}

In 2014, a repeating sequence of Glu-Ala-Leu-Ala, GALA and exosomes were combined by Nakase and Futaki for cytosolic delivery. As a cationic lipid, GALA is a $\mathrm{pH}$-sensitive peptide with an amphiphilic structure and higher efficiency (Liu et al., 2015). The GALA peptide was intrinsically applied with cationic lipids to achieve the cytosolic delivery of peptides and proteins, and it also interacts with cell membranes to enable viral gene escape from acidic endosomes using a mimicking effect (Chen W. et al., 2018).

\section{Dual Ligand-Mediated Exosome Engineering}

Dual ligand-mediated endocytosis can be applied for inducing the accumulation of exosomes at specific target sites. The permeability of the exosome membrane can be reduced to enhance the release of drugs that inhibit tumor development, enabling precise cancer therapy applications in the future.

Recent research has emphasized attempts to overcome nonsmall cell lung cancer (NSCLC), the predominant type of lung cancer (Liu et al., 2018), by exosome engineering strategy. The modification with polyethylene glycol-aminoethyl anisamide (PEG-AA) could acted as the dual legend to enhance the exosome circulation time and allow for the targeting of pulmonary metastatic tissues with sigma receptor overexpression. By using this modified exosome loaded with PTX and PEG-AA, the specific drug can be delivered to target cells with high loading capacity (Kim et al., 2018).

\section{Engineering Exosomes by Fusion With Liposomes}

As a superior drug delivery vehicle, liposomes can load drugs within the aqueous compartment and concentric lipid bilayers, improving the therapeutic efficiency for pharmaceuticals, and vaccines (Bozzuto and Molinari, 2015).

Sato et al. applied the freeze-thaw method to help the fusion of liposomes with exosomal membrane. The delivery efficiency was significantly optimized because of the decreased immunogenicity of the exosome surface and increased colloidal stability with higher circulation time. The study demonstrated a new technology for engineering hybrid exosomes as bionanotransporters, which can be used for hydrophilic cargos to recipient cells by the specific fusion strategy (Sato et al., 2016).

\section{Exosome-Coated Metal-Organic Framework Nanoparticles}

Metal-organic frameworks (MOFs) constitute a class of crystalline materials with diverse modularity, high crystallinity, 


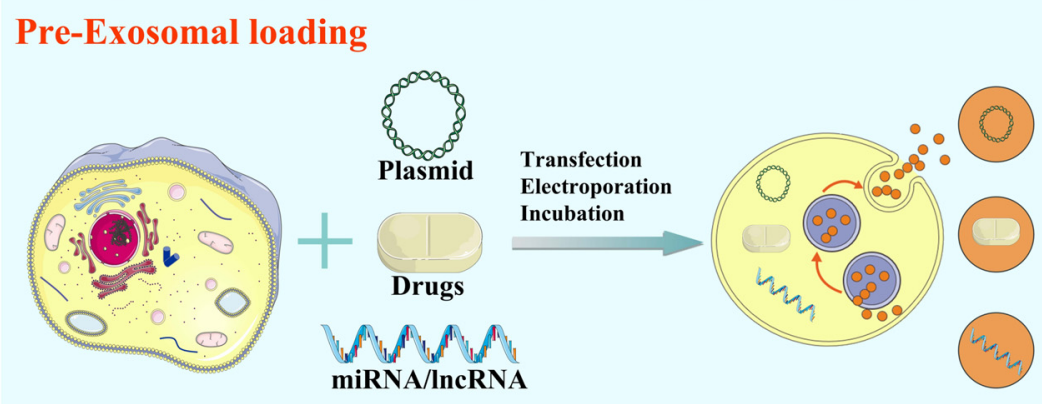

Post-Exosomal loading

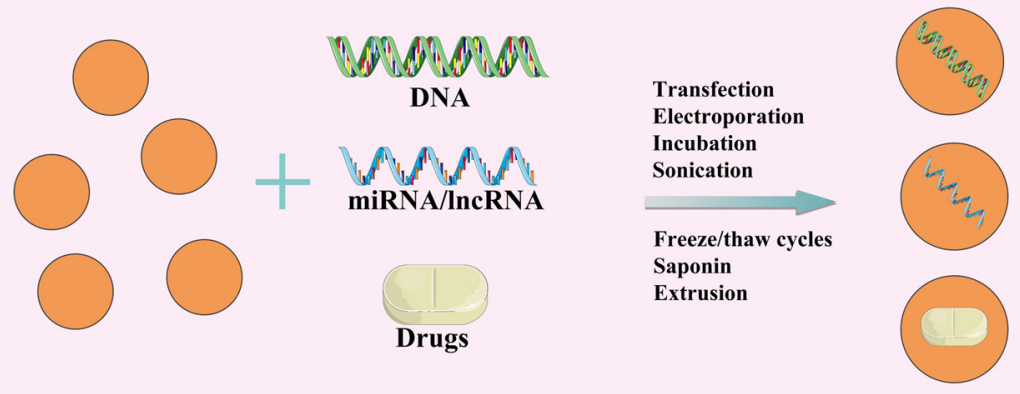

Specific loading strategies (Pre-loading)

Overexpression of cytoplasmic proteins
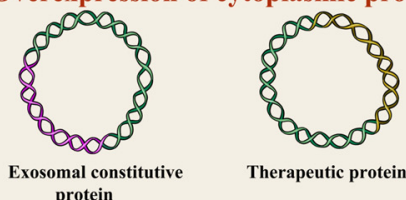

Therapeutic protein

Fusion of target protein

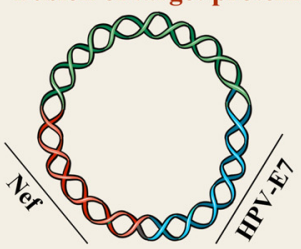

Intrinsic modifiction

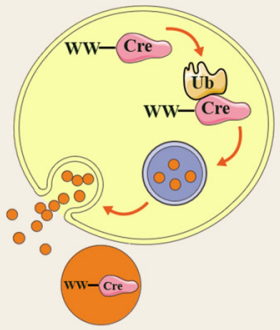

Guidance of proteins and sequences

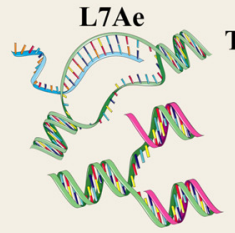

Translational regulation

Box C/D Kink-turn

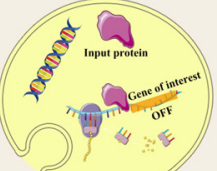

CRISPR/Cas9

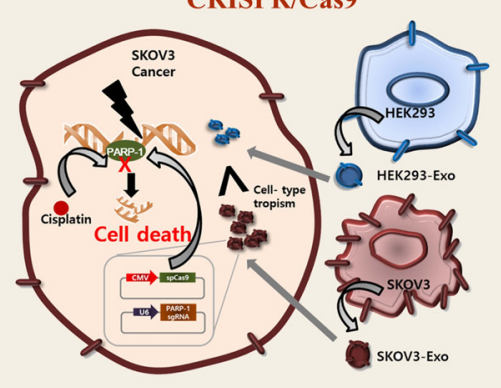

FIGURE 2 | Design strategies for the modifications of exosomal surface. Multiple strategies to be done before exosomes can be successfully translated into new technologies to improve the targeting ability of donor cells and therapeutic efficacy of chemical and biomolecular drugs. 
exceptional porosity, and intriguing architectures that can be employed in gas storage, purification, and separation, as well used for catalysis and sensing applications (Zhou et al., 2018). MOFs consist of one-, two-, or three-dimensional (1D, 2D, or 3D) structural topologies as organic-inorganic hybrids with tight junction between transition-metal cations and multidentate organic linkers (Liu et al., 2016). Therefore, MOFs can be applied in nanomedicine due to sufficient loading capacity, controlled drug-release properties, and intrinsic biodegradability (Al Haydar et al., 2017).

In 2017, Illes et al. combined exosomes with MOFs to generate a smart and efficient drug carrier with an "onboard trigger." The features of MOF NPs and exosomes were intrinsically combined, facilitating efficient and straightforward loading and retention of the cargo. Moreover, for intracellular release, the cargo was decomposed into substances mediated by endogenous exosomal release mechanisms and intrinsic biodegradability, showing great deliverability without premature leakage (Illes et al., 2017).

\section{DISCUSSION}

Since exosomes were first designed for drug delivery by leveraging the ability of mRNAs and miRNAs (Valadi et al., 2007), exosomes have been widely applied for the delivery system with multiple superiority, such as specificity to target tissues, stability, a long-circulating half-life, and biocompatibility. However, several essential factors still need to be considered for further application:

(i) Homing sources. Raw 264.7 macrophages, dendritic cells, and mesenchymal stromal cells are the most common sources for the exosomes, which could significantly affect the specific content and biological performance. The specific roles of various exosomes originated from different cells are essential for further research. For example, the macrophagederived exosomes could easily across $\mathrm{BBB}$ without any modifications for drug delivery. The exosomes derived from rhabdomyosarcoma (RMS) cell, osteoclast and bronchial fibroblast can be applied for specifically targeting fibroblast cells, osteoblast cells and epithelial targets. Additionally, the exosomes from malignant mesothelioma (MM) cells, metastatic cancer cells and pancreatic cancer cells (PCC) could directly targeting cancer cells for cancer treatments. It is great potential to choose appropriate homing sources for further studies.

(ii) Isolation methodology. Ultracentrifugation and filtration are the most common strategies for the isolation of exosomes. The previously reported isolation methods could not efficiently be applied in the fields of therapeutics with various disadvantages, including low yield, reduced purity, disrupted structure, and incomplete properties. Furthermore, a great challenge still existed for separating exosomes contaminated with various substances, including liposome, protein and RNA. On this issue, Möller et al. found that size-exclusion chromatography (SEC) combined with ultrafiltration was fast and efficient with highly purity and integrity for isolation approaches (Lobb et al., 2015), which is a strategy measured by the efficiency penetrating the stationary phase based on the size of different compounds. Moreover, an immunomagnetic strategy reported by Pedersen et al. could efficiently obtain ultrapure exosomes by targeting exosomal markers (Li et al., 2017). Polymer precipitation were also applied to isolate and purify polymers based on the formation of meshlike net, with the advantage in the detection of biomarkers in vesicles (Deregibus et al., 2016; Figure 3). A standardized and

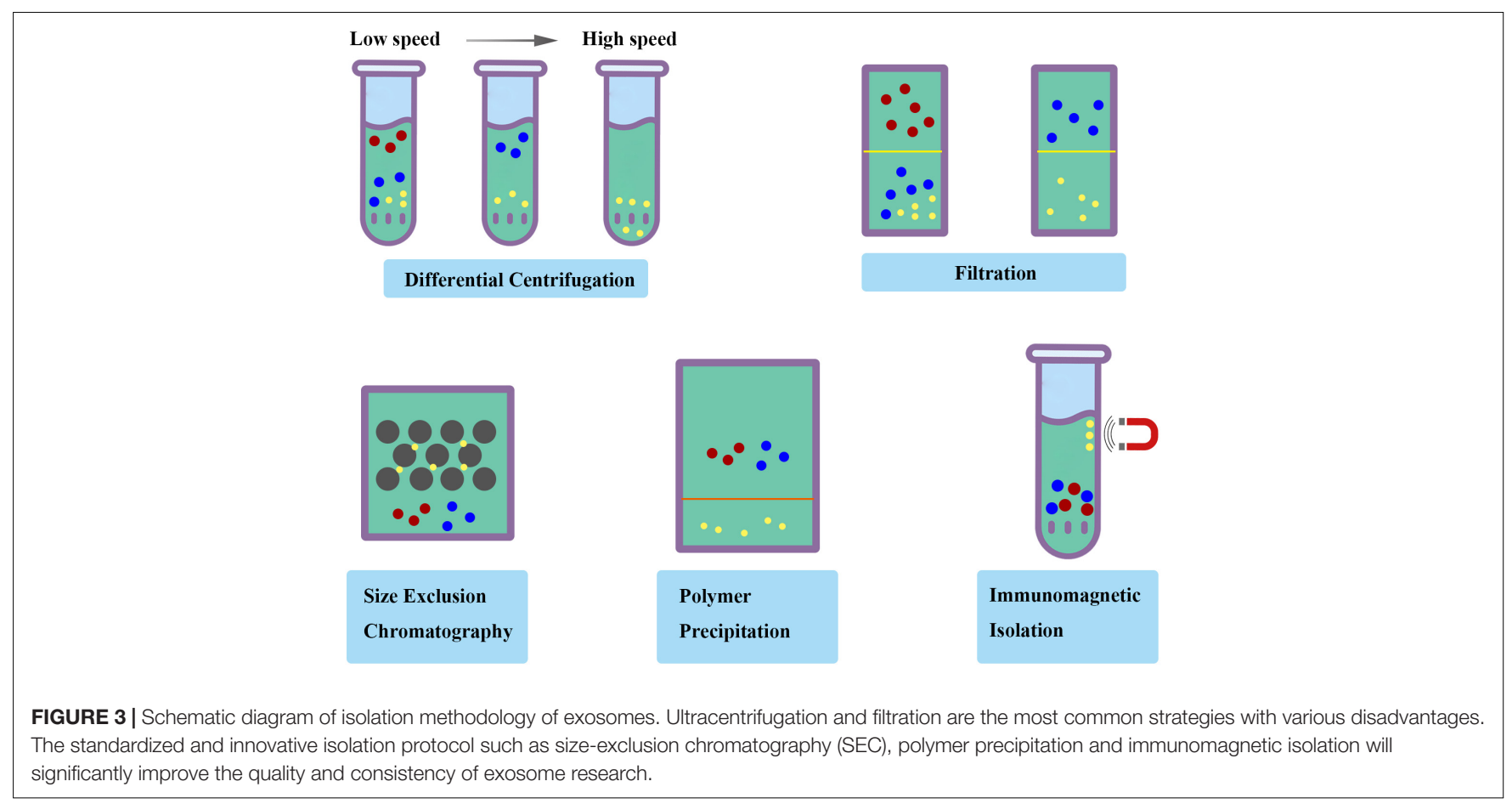


innovative isolation protocol will significantly improve the quality and consistency of exosome research.

(iii) Loading strategies for engineered exosomes. Several mechanical methods, including mechanical extrusion, electroporation, saponin permeabilization, incubation at room temperature, repeated freeze-thaw cycles, and sonication were widely verified. The purification of exosomes remains laborious, and the integrity and biological activity of exosomes are incompletely resolved with some technical difficulties depending on these conventional methods. The appropriate choice and newly designed advances were essential for the loading strategy. For example, comparing the multiple loading methods for paclitaxel, such as pure incubation, mixing, electroporation, and sonication, the loading efficiency was found to be enhanced by approximately 30\%, without compromised stability or functionality of the exosomal formulation (Kim et al., 2016). Moreover, exosomes loaded with magnetic nanoparticles can be targeted to external magnetic field, including the superparamagnetic nanoparticle and iron oxide. Therefore, the large-scale production of engineered exosomes can be explored and expanded with higher loading efficiency and accurate positioning.

(iv) Modification of exosomes for drug delivery. The surface modification, fusion proteins, dual legend, and fusion with liposomes have been currently applied for the method of the modification (Alvarez-Erviti et al., 2011; Ohno et al., 2013; Bellavia et al., 2017). Despite ever-deeper understanding, the advances of modification of exosomes are essential for the improvement of the clinical translation (Jia et al., 2018; Tian et al., 2018). The delicate drug delivery strategy targeting to a particular tissue or a specific type of cell could avoid decentralized distribution to other tissues and preventing degradation by immune response (Wen et al., 2016). Target T cells, cell receptors, cell adhesion molecules and antigen presentation are four main type for surface modification. The cell-penetrating peptides and T7, a transferrin receptor-building peptide were reported recently for surface modification to enhance targeting ability. Furthermore, the low level long-term accumulation in organs and

\section{REFERENCES}

Agrawal, A. K., Aqil, F., Jeyabalan, J., Spencer, W. A., Beck, J., Gachuki, B. W., et al. (2017). Milk-derived exosomes for oral delivery of paclitaxel. Nanomedicine 13, 1627-1636. doi: 10.1016/j.nano.2017.03.001

Al Haydar, M., Abid, H. R., Sunderland, B., and Wang, S. (2017). Metal organic frameworks as a drug delivery system for flurbiprofen. Drug Des. Devel. Ther. 11, 2685-2695. doi: 10.2147/dddt.s145716

Alenquer, M., and Amorim, M. J. (2015). Exosome biogenesis, regulation, and function in viral infection. Viruses 7, 5066-5083. doi: 10.3390/v7092862

Alvarez-Erviti, L., Seow, Y., Yin, H., Betts, C., Lakhal, S., and Wood, M. J. (2011). Delivery of siRNA to the mouse brain by systemic injection of targeted exosomes. Nat. Biotechnol. 29, 341-345. doi: 10.1038/nbt.1807

Barile, L., and Vassalli, G. (2017). Exosomes: therapy delivery tools and biomarkers of diseases. Pharmacol. Ther. 174, 63-78. doi: 10.1016/j.pharmthera.2017. 02.020

Batagov, A. O., Kuznetsov, V. A., and Kurochkin, I. V. (2011). Identification of nucleotide patterns enriched in secreted RNAs as putative cis-acting elements tissues with concomitant low systemic toxicity is also facilitated by cellular uptake. Therefore, modifications to the exosomal surface and components of the donor cells need to be studied for the improvement of targeting strategies. It must also be clear how to convert theoretical research tinto clinical contributions with large-scale prospective studies.

\section{CONCLUSION}

The past decade has witnessed confluent advances in the knowledge of exosomes for next-generation diagnostics, disease surveillance, and individual diagnosis and therapy, which was widely applied for early diagnosis and delivery system with higher efficacy. Further advanced and improvements for the drug loading strategies and modification methods will be favor of the clinical translation in the future.

\section{AUTHOR CONTRIBUTIONS}

YW and XS proposed the conception for the review. MX and QY wrote the manuscript and prepared the figures and table. YW and MX gave critical discussions and revisions on manuscript. All authors read and approved the final manuscript.

\section{FUNDING}

This research was funded by the China National Key Research and Development Program Stem Cell and Translational Research Key Projects (2018YFA0108301), National Natural Science Foundation of China (31971109, 31471390), Shanghai RisingStar Program (17QA1405400), Shanghai Health and Family Planning System Program (2017YQ028), National Major Scientific and Technological Special Project for "Significant New Drugs Development" during the Thirtieth Five-year Plan Period (2019ZX09301113), and Science and Technology Commission of Shanghai Municipality (19495800700).

targeting them to exosome nano-vesicles. BMC Genomics 12(Suppl. 3):S18. doi: 10.1186/1471-2164-12-S3-S18

Bellavia, D., Raimondo, S., Calabrese, G., Forte, S., Cristaldi, M., Patinella, A., et al. (2017). Interleukin 3- receptor targeted exosomes inhibit in vitro and in vivo Chronic Myelogenous Leukemia cell growth. Theranostics 7, 1333-1345. doi: 10.7150/thno.17092

Bonoiu, A. C., Mahajan, S. D., Ding, H., Roy, I., Yong, K. T., Kumar, R., et al. (2009). Nanotechnology approach for drug addiction therapy: gene silencing using delivery of gold nanorod-siRNA nanoplex in dopaminergic neurons. Proc. Natl. Acad. Sci. U.S.A. 106, 5546-5550. doi: 10.1073/pnas.0901715106

Bozzuto, G., and Molinari, A. (2015). Liposomes as nanomedical devices. Int. J. Nanomedicine 10, 975-999. doi: 10.2147/ijn.s68861

Bunggulawa, E. J., Wang, W., Yin, T., Wang, N., Durkan, C., Wang, Y., et al. (2018). Recent advancements in the use of exosomes as drug delivery systems. J. Nanobiotechnol. 16:81.

Chen, G., Huang, A. C., Zhang, W., Zhang, G., Wu, M., Xu, W., et al. (2018). Exosomal PD-L1 contributes to immunosuppression and is associated with anti-PD-1 response. Nature 560, 382-386. 
Chen, W., Yang, M., Bai, J., Li, X., Kong, X., Gao, Y., et al. (2018). Exosomemodified tissue engineered blood vessel for endothelial progenitor cell capture and targeted siRNA delivery. Macromol. Biosci. 18:1700242. doi: 10.1002/mabi. 201700242

Cheng, Y., and Schorey, J. S. (2016). Targeting soluble proteins to exosomes using a ubiquitin tag. Biotechnol. Bioeng. 113, 1315-1324. doi: 10.1002/bit.25884

De Rosa, G., Salzano, G., Caraglia, M., and Abbruzzese, A. (2012). Nanotechnologies: a strategy to overcome blood-brain barrier. Curr. Drug Metab. 13, 61-69. doi: 10.2174/138920012798356943

Deregibus, M. C., Figliolini, F., D’Antico, S., Manzini, P. M., Pasquino, C., De Lena, M., et al. (2016). Charge-based precipitation of extracellular vesicles. Int. J. Mol. Med. 38, 1359-1366.

Hall, J., Prabhakar, S., Balaj, L., Lai, C. P., Cerione, R. A., and Breakefield, X. O. (2016). Delivery of therapeutic proteins via extracellular vesicles: review and potential treatments for Parkinson's disease, Glioma, and Schwannoma. Cell Mol. Neurobiol. 36, 417-427. doi: 10.1007/s10571-015-0309-0

Haney, M. J., Klyachko, N. L., Zhao, Y., Gupta, R., Plotnikova, E. G., He, Z., et al. (2015). Exosomes as drug delivery vehicles for Parkinson's disease therapy. J. Control Release 207, 18-30.

Heinemann, M. L., and Vykoukal, J. (2017). Sequential filtration: a gentle method for the isolation of functional extracellular vesicles. Methods Mol. Biol. 1660, 33-41. doi: 10.1007/978-1-4939-7253-1_4

Illes, B., Hirschle, P., Barnert, S., Cauda, V., Wuttke, S., and Engelke, M. (2017). Exosome-coated metal-organic framework nanoparticles: an efficient drug delivery platform. Chem. Mater. 29, 8042-8046. doi: 10.1021/acs.chemmater. $7 \mathrm{~b} 02358$

Jia, G., Han, Y., An, Y., Ding, Y., He, C., Wang, X., et al. (2018). NRP-1 targeted and cargo-loaded exosomes facilitate simultaneous imaging and therapy of glioma in vitro and in vivo. Biomaterials 178, 302-316. doi: 10.1016/j.biomaterials. 2018.06.029

Kamerkar, S., LeBleu, V. S., Sugimoto, H., Yang, S., Ruivo, C. F., Melo, S. A., et al. (2017). Exosomes facilitate therapeutic targeting of oncogenic KRAS in pancreatic cancer. Nature 546, 498-503. doi: 10.1038/nature22341

Kim, M. S., Haney, M. J., Zhao, Y., Mahajan, V., Deygen, I., Klyachko, N. L., et al. (2016). Development of exosome-encapsulated paclitaxel to overcome MDR in cancer cells. Nanomedicine 12, 655-664. doi: 10.1016/j.nano.2015.10.012

Kim, M. S., Haney, M. J., Zhao, Y., Yuan, D., Deygen, I., Klyachko, N. L., et al. (2018). Engineering macrophage-derived exosomes for targeted paclitaxel delivery to pulmonary metastases: in vitro and in vivo evaluations. Nanomedicine 14, 195-204. doi: 10.1016/j.nano.2017.09.011

Kim, S. M., Yang, Y., Oh, S. J., Hong, Y., Seo, M., and Jang, M. (2017). Cancerderived exosomes as a delivery platform of CRISPR/Cas 9 confer cancer cell tropism-dependent targeting. J. Control Release 266, 8-16. doi: 10.1016/j. jconrel.2017.09.013

Kojima, R., Bojar, D., Rizzi, G., Hamri, G. C., El-Baba, M. D., Saxena, P., et al. (2018). Designer exosomes produced by implanted cells intracerebrally deliver therapeutic cargo for Parkinson's disease treatment. Nat. Commun. 9:1305.

Lamichhane, T. N., Raiker, R. S., and Jay, S. M. (2015). Exogenous DNA loading into extracellular vesicles via electroporation is size-dependent and enables limited gene delivery. Mol. Pharm. 12, 3650-3657. doi: 10.1021/acs. molpharmaceut.5b00364

Lee, H., Park, H., Noh, G. J., and Lee, E. S. (2018). pH-responsive hyaluronateanchored extracellular vesicles to promote tumor-targeted drug delivery. Carbohydr. Polym. 202, 323-333. doi: 10.1016/j.carbpol.2018.08.141

Li, P., Kaslan, M., Lee, S. H., Yao, J., and Gao, Z. (2017). Progress in exosome isolation techniques. Theranostics 7, 789-804. doi: 10.7150/thno.18133

Li, S. P., Lin, Z. X., Jiang, X. Y., and Yu, X. Y. (2018). Exosomal cargo-loading and synthetic exosome-mimics as potential therapeutic tools. Acta Pharmacol. Sin. 39, 542-551. doi: 10.1038/aps.2017.178

Liew, L. C., Katsuda, T., Gailhouste, L., Nakagama, H., and Ochiya, T. (2017). Mesenchymal stem cell-derived extracellular vesicles: a glimmer of hope in treating Alzheimer's disease. Int. Immunol. 29, 11-19. doi: 10.1093/intimm/ dxx002

Lin, Y., Wu, J., Gu, W., Huang, Y., Tong, Z., Huang, L., et al. (2018). Exosomeliposome hybrid nanoparticles deliver CRISPR/Cas9 system in MSCs. Adv. Sci. 5:1700611. doi: $10.1002 /$ advs.201700611

Liu, C., and Su, C. (2019). Design strategies and application progress of therapeutic exosomes. Theranostics 9, 1015-1028. doi: 10.7150/thno.30853
Liu, R., Wang, X., Curtiss, C., Landas, S., Rong, R., Sheikh, M. S., et al. (2018). Monoglyceride lipase gene knockout in mice leads to increased incidence of lung adenocarcinoma. Cell Death Dis. 9:36.

Liu, R., Yu, T., Shi, Z., and Wang, Z. (2016). The preparation of metal-organic frameworks and their biomedical application. Int. J. Nanomedicine 11, 11871200. doi: 10.2147/ijn.s100877

Liu, Y., Li, D., Liu, Z., Zhou, Y., Chu, D., Li, X., et al. (2015). Targeted exosomemediated delivery of opioid receptor $\mathrm{Mu}$ siRNA for the treatment of morphine relapse. Sci. Rep. 5:17543.

Lobb, R. J., Becker, M., Wen, S. W., Wong, C. S., Wiegmans, A. P., Leimgruber, A., et al. (2015). Optimized exosome isolation protocol for cell culture supernatant and human plasma. J. Extracell. Vesicles 4:27031. doi: 10.3402/jev.v4.27031

Luo, Q., Guo, D., Liu, G., Chen, G., Hang, M., and Jin, M. (2017). Exosomes from MiR-126-Overexpressing Adscs are therapeutic in relieving acute myocardial ischaemic injury. Cell Physiol. Biochem. 44, 2105-2116. doi: 10.1159/000485949

Manfredi, F., Di Bonito, P., Arenaccio, C., Anticoli, S., and Federico, M. (2016). Incorporation of heterologous proteins in engineered exosomes. Methods Mol. Biol. 1448, 249-260. doi: 10.1007/978-1-4939-3753-0_18

Niu, Z., Pang, R. T. K., Liu, W., Li, Q., Cheng, R., and Yeung, W. S. B. (2017). Polymer-based precipitation preserves biological activities of extracellular vesicles from an endometrial cell line. PLoS One 12:e0186534. doi: 10.1371/ journal.pone.0186534

Ohno, S., Takanashi, M., Sudo, K., Ueda, S., Ishikawa, A., Matsuyama, N., et al. (2013). Systemically injected exosomes targeted to EGFR deliver antitumor microRNA to breast cancer cells. Mol. Ther. 21, 185-191. doi: 10.1038/mt. 2012.180

Park, E. J., Prajuabjinda, O., Soe, Z. Y., Darkwah, S., Appiah, M. G., Kawamoto, E., et al. (2019). Exosomal regulation of lymphocyte homing to the gut. Blood Adv. 3, 1-11. doi: 10.1182/bloodadvances.2018024877

Pascucci, L., Cocce, V., Bonomi, A., Ami, D., Ceccarelli, P., Ciusani, E., et al. (2014). Paclitaxel is incorporated by mesenchymal stromal cells and released in exosomes that inhibit in vitro tumor growth: a new approach for drug delivery. J. Control Release 192, 262-270. doi: 10.1016/j.jconrel.2014.07.042

Qi, H., Liu, C., Long, L., Ren, Y., Zhang, S., Chang, X., et al. (2016). Blood exosomes endowed with magnetic and targeting properties for cancer therapy. ACS Nano 10, 3323-3333. doi: 10.1021/acsnano.5b06939

Qu, M., Lin, Q., Huang, L., Fu, Y., Wang, L., He, S., et al. (2018). Dopamine-loaded blood exosomes targeted to brain for better treatment of Parkinson's disease. J. Control Release 287, 156-166. doi: 10.1016/j.jconrel.2018.08.035

Saari, H., Lazaro-Ibanez, E., Viitala, T., Vuorimaa-Laukkanen, E., Siljander, P., and Yliperttula, M. (2015). Microvesicle- and exosome-mediated drug delivery enhances the cytotoxicity of Paclitaxel in autologous prostate cancer cells. J. Control Release 220(Pt B), 727-737. doi: 10.1016/j.jconrel.2015.09.031

Saito, H., Kobayashi, T., Hara, T., Fujita, Y., Hayashi, K., Furushima, R., et al. (2010). Synthetic translational regulation by an L7Ae-kink-turn RNP switch. Nat. Chem. Biol. 6, 71-78. doi: 10.1038/nchembio.273

Sato, Y. T., Umezaki, K., Sawada, S., Mukai, S. A., Sasaki, Y., Harada, N., et al. (2016). Engineering hybrid exosomes by membrane fusion with liposomes. Sci. Rep. 6:21933.

Shtam, T. A., Kovalev, R. A., Varfolomeeva, E. Y., Makarov, E. M., Kil, Y. V., and Filatov, M. V. (2013). Exosomes are natural carriers of exogenous siRNA to human cells in vitro. Cell Commun. Signal. 11:88. doi: 10.1186/1478-811x$11-88$

Skog, J., Wurdinger, T., van Rijn, S., Meijer, D. H., Gainche, L., Sena-Esteves, M., et al. (2008). Glioblastoma microvesicles transport RNA and proteins that promote tumour growth and provide diagnostic biomarkers. Nat. Cell Biol. 10, 1470-1476. doi: 10.1038/ncb1800

Sterzenbach, U., Putz, U., Low, L. H., Silke, J., Tan, S. S., and Howitt, J. (2017). Engineered exosomes as vehicles for biologically active proteins. Mol. Ther. 25, 1269-1278. doi: 10.1016/j.ymthe.2017.03.030

Sun, D., Zhuang, X., Xiang, X., Liu, Y., Zhang, S., Liu, C., et al. (2010). A novel nanoparticle drug delivery system: the anti-inflammatory activity of curcumin is enhanced when encapsulated in exosomes. Mol. Ther. 18, 1606-1614. doi: 10.1038/mt.2010.105

Tian, T., Zhang, H. X., He, C. P., Fan, S., Zhu, Y. L., Qi, C., et al. (2018) Surface functionalized exosomes as targeted drug delivery vehicles for cerebral ischemia therapy. Biomaterials 150, 137-149. doi: 10.1016/j.biomaterials.2017. 10.012 
Tian, Y., Li, S., Song, J., Ji, T., Zhu, M., Anderson, G. J., et al. (2014). A doxorubicin delivery platform using engineered natural membrane vesicle exosomes for targeted tumor therapy. Biomaterials 35, 2383-2390. doi: 10 . 1016/j.biomaterials.2013.11.083

Tran, P. H. L., Xiang, D., Tran, T. T. D., Yin, W., Zhang, Y., Kong, L., et al. (2019). Exosomes and nanoengineering: a match made for precision therapeutics. $A d v$. Mater. 32:e1904040.

Valadi, H., Ekstrom, K., Bossios, A., Sjostrand, M., Lee, J. J., and Lotvall, J. O. (2007). Exosome-mediated transfer of mRNAs and microRNAs is a novel mechanism of genetic exchange between cells. Nat. Cell Biol. 9, 654-659. doi: 10.1038/ncb1596

Vinas, J. L., Burger, D., Zimpelmann, J., Haneef, R., Knoll, W., Campbell, P., et al. (2016). Transfer of microRNA-486-5p from human endothelial colony forming cell-derived exosomes reduces ischemic kidney injury. Kidney Int. 90, 1238-1250. doi: 10.1016/j.kint.2016.07.015

Wahlgren, J., De, L., Karlson, T., Brisslert, M., Vaziri Sani, F., Telemo, E., et al. (2012). Plasma exosomes can deliver exogenous short interfering RNA to monocytes and lymphocytes. Nucleic Acids Res. 40:e130. doi: 10.1093/nar/ gks463

Wang, X., Zhang, H., Bai, M., Ning, T., Ge, S., Deng, T., et al. (2018). Exosomes serve as nanoparticles to deliver anti-miR-214 to reverse chemoresistance to cisplatin in gastric cancer. Mol. Ther. 26, 774-783. doi: 10.1016/j.ymthe.2018. 01.001

Wang, Y., Chen, X., Tian, B., Liu, J., Yang, L., Zeng, L., et al. (2017). Nucleolintargeted extracellular vesicles as a versatile platform for biologics delivery to breast cancer. Theranostics 7, 1360-1372. doi: 10.7150/thno.16532

Wen, S. W., Sceneay, J., Lima, L. G., Wong, C. S., Becker, M., Krumeich, S., et al. (2016). The biodistribution and immune suppressive effects of breast cancerderived exosomes. Cancer Res. 76, 6816-6827. doi: 10.1158/0008-5472.can-160868

Yang, J., Zhang, X., Chen, X., Wang, L., and Yang, G. (2017). Exosome mediated delivery of miR-124 promotes neurogenesis after ischemia. Mol. Ther. Nucleic Acids 7, 278-287. doi: 10.1016/j.omtn.2017.04.010
Yuan, D., Zhao, Y., Banks, W. A., Bullock, K. M., Haney, M., Batrakova, E., et al. (2017). Macrophage exosomes as natural nanocarriers for protein delivery to inflamed brain. Biomaterials 142, 1-12. doi: 10.1016/j.biomaterials.2017.07.011

Zarovni, N., Corrado, A., Guazzi, P., Zocco, D., Lari, E., Radano, G., et al. (2015). Integrated isolation and quantitative analysis of exosome shuttled proteins and nucleic acids using immunocapture approaches. Methods 87, 46-58. doi: 10. 1016/j.ymeth.2015.05.028

Zhang, H., Wang, Y., Bai, M., Wang, J., Zhu, K., Liu, R., et al. (2018). Exosomes serve as nanoparticles to suppress tumor growth and angiogenesis in gastric cancer by delivering hepatocyte growth factor siRNA. Cancer Sci. 109, 629-641. doi: $10.1111 /$ cas. 13488

Zhou, Y., Tian, T., Zhu, Y., Jaffar Ali, D., Hu, F., Qi, Y., et al. (2017). Exosomes transfer among different species cells and mediating miRNAs Delivery. J. Cell. Biochem. 118, 4267-4274. doi: 10.1002/jcb.26077

Zhou, Z., Xing, X., Tian, C., Wei, W., Li, D., Hu, F., et al. (2018). A multifunctional nanocage-based MOF with Tri- and Tetranuclear Zinc cluster secondary building units. Sci. Rep. 8:3117.

Zhuang, X., Xiang, X., Grizzle, W., Sun, D., Zhang, S., Axtell, R. C., et al. (2011). Treatment of brain inflammatory diseases by delivering exosome encapsulated anti-inflammatory drugs from the nasal region to the brain. Mol. Ther. 19, 1769-1779. doi: 10.1038/mt.2011.164

Conflict of Interest: The authors declare that the research was conducted in the absence of any commercial or financial relationships that could be construed as a potential conflict of interest.

Copyright (c) $2020 \mathrm{Xu}$, Yang, Sun and Wang. This is an open-access article distributed under the terms of the Creative Commons Attribution License (CC BY). The use, distribution or reproduction in other forums is permitted, provided the original author(s) and the copyright owner(s) are credited and that the original publication in this journal is cited, in accordance with accepted academic practice. No use, distribution or reproduction is permitted which does not comply with these terms. 